\title{
Full Core Subcritical Multiplication Ex-Core Calculations with Shift
}

Katherine Royston, ORNL Tara Pandya, ORNL

May 12, 2020
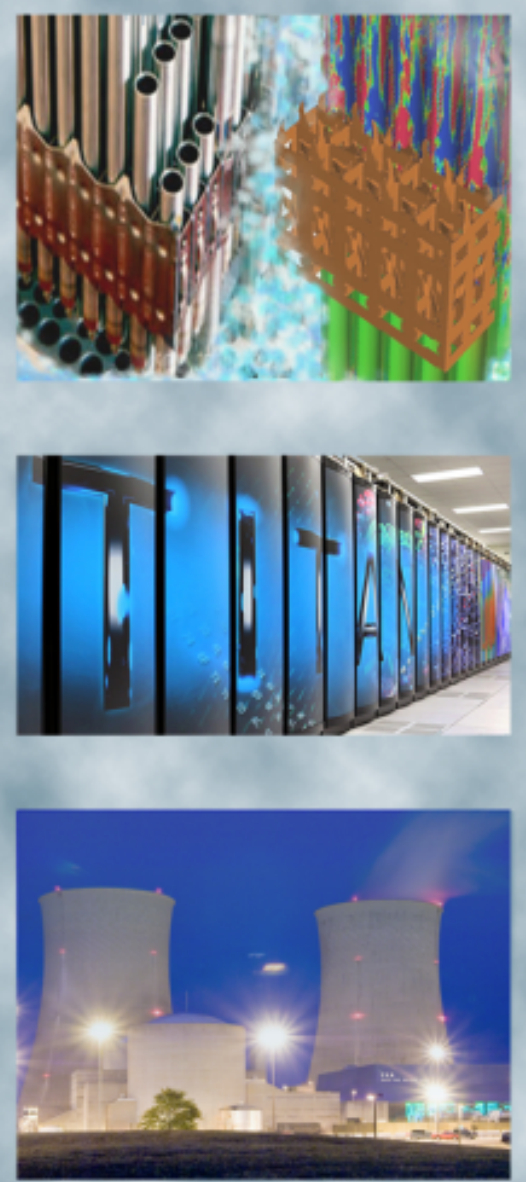


\title{
DOCUMENT AVAILABILITY
}

Reports produced after January 1, 1996, are generally available free via US Department of Energy (DOE) SciTech Connect.

Website www.osti.gov

Reports produced before January 1, 1996, may be purchased by members of the public from the following source:

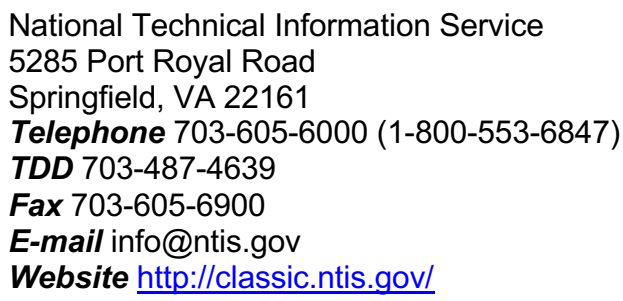

Reports are available to DOE employees, DOE contractors, Energy Technology Data Exchange representatives, and International Nuclear Information System representatives from the following source:

Office of Scientific and Technical Information

PO Box 62

Oak Ridge, TN 37831

Telephone 865-576-8401

Fax 865-576-5728

E-mail reports@osti.gov

Website http://www.osti.gov/contact.html

\begin{abstract}
This report was prepared as an account of work sponsored by an agency of the United States Government. Neither the United States Government nor any agency thereof, nor any of their employees, makes any warranty, express or implied, or assumes any legal liability or responsibility for the accuracy, completeness, or usefulness of any information, apparatus, product, or process disclosed, or represents that its use would not infringe privately owned rights. Reference herein to any specific commercial product, process, or service by trade name, trademark, manufacturer, or otherwise, does not necessarily constitute or imply its endorsement, recommendation, or favoring by the United States Government or any agency thereof. The views and opinions of authors expressed herein do not necessarily state or reflect those of the United States Government or any agency thereof.
\end{abstract}


REVISION LOG

\begin{tabular}{|c|c|c|c|}
\hline Revision & Date & Affected Pages & Revision Description \\
\hline 0 & July 14, 2020 & All & Initial Public Release - Unlimited \\
\hline & & & \\
\hline & & & \\
\hline & & & \\
\hline
\end{tabular}

\section{Document pages that are:}

Export Controlled ___ None

IP/Proprietary/NDA Controlled___ None

Sensitive Controlled___ None

\section{Requested Distribution:}

To:

Bill Martin

Dave Kropaczek

Copy:

William Cramer 


\section{EXECUTIVE SUMMARY}

This report details the development and testing of Virtual Environment for Reactor Applications (VERA) as applied to full core subcritical multiplication ex-core calculations with Shift. The limiting factor in being able to run these detector calculations with Monte Carlo is the amount of computational memory used by a fully-loaded full core problem. The main development features added to VERA in this effort were enabling source biasing and separable weight windows and integrating multithreading in Shift. With these capabilities, a subcritical multiplication detector response calculation on a full core Watts Bar Nuclear Plant Unit 1 (WBN1) ex-core model was successfully run on a moderately sized computing cluster. 


\section{CONTENTS}

EXECUTIVE SUMMARY $\ldots \ldots \ldots \ldots \ldots \ldots \ldots \ldots$ iv

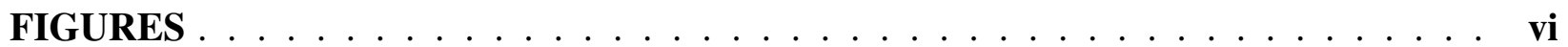

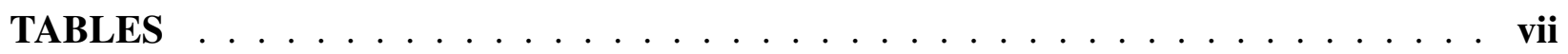

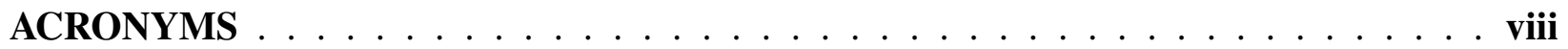

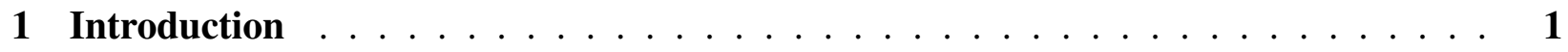

2 Methodology . . . . . . . . . . . . . . . . . . . . 2

2.1 Source Biasing and Weight Windows . . . . . . . . . . . . . . . 2

2.2 Fission Source Spectrum . . . . . . . . . . . . . . . . . . 2

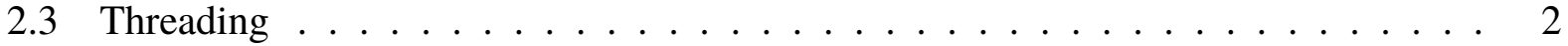

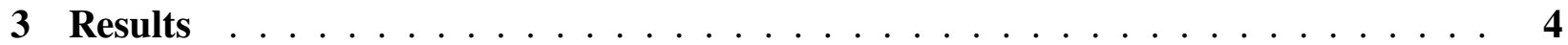

3.1 Fission Source Spectrum . . . . . . . . . . . . . . . . . 4

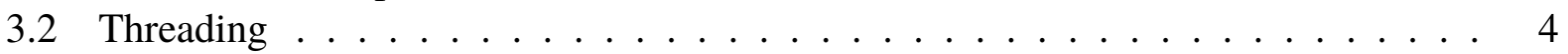

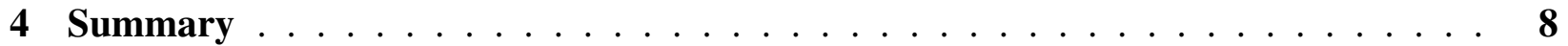

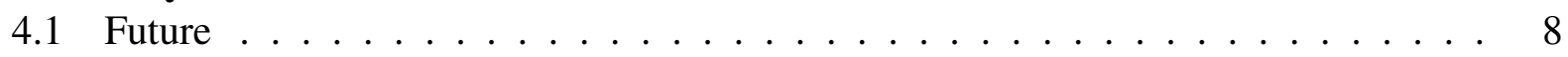

5 Acknowledgments $\ldots \ldots \ldots \ldots \ldots \ldots \ldots \ldots \ldots \ldots \ldots$ 


\section{FIGURES}

1 Axial slice of the WBN1 model at an elevation of $200 \mathrm{~cm} . \quad \ldots \ldots$

2 Axial slice of the WBN1 adjoint function for the north and south source range detectors at an elevation of $200 \mathrm{~cm} \ldots \ldots \ldots \ldots$

3 Spatial distributions of the nuclide sources transferred from MPACT to Shift . . . . 6 


\section{TABLES}

1 Computation time for WBN1 full core subcritical model. . . . . . . . . . . . 7 


\section{ACRONYMS}

CADES Compute and Data Environment for Science

CADIS Consistent Adjoint Driven Importance Sampling

CTF COBRA-TF

HPC high performance computing

MC Monte Carlo

MPI message passing interface

ORNL Oak Ridge National Laboratory

TVA Tennessee Valley Authority

VERA Virtual Environment for Reactor Applications

WBN1 Watts Bar Nuclear Plant Unit 1 


\section{INTRODUCTION}

This report details the development and testing completed under milestone L3.RTM.MCH.P19.01. The goal of this milestone was to enable full core subcritical multiplication ex-core calculations through Virtual Environment for Reactor Applications (VERA) using Shift. The limiting factor in being able to run these calculations on modern day computing clusters and high performance computing (HPC) machines is the large amount of memory required for Consistent Adjoint Driven Importance Sampling (CADIS) and the Monte Carlo (MC) part of the simulation.

The execution plan for this milestone was the following:

1. Integrate source biasing and new separable weight windows,

2. Investigate using MPACT energy spectrum,

3. Implement memory usage improvements as part of milestone L2.RTM.MCH.P19.01,

4. Run Shift full core subcritical multiplication calculation, and

5. Document work performed and calculation results in a technical note.

This report completes item 5 above. The remainder of this report describes the basic development tasks completed (items 1-3 above) in Sec. 2, followed by the testing results (item 4 above) in Section 3. This report concludes with a summary and future planned work in continuing projects presented in Section 4. 


\section{METHODOLOGY}

\subsection{SOURCE BIASING AND WEIGHT WINDOWS}

Integration of full source biasing from Shift into VERA is complete. This integration was needed in this milestone to fully implement the CADIS method for variance reduction [1]. Along with this, the abilities to use separable weight windows and an updated variance reduction tracking in Shift were incorporated. Separable weight windows can reduce the amount of memory storage required for the weight windows used for CADIS by separating the energy and spatial domains [2]. The updated variance reduction tracking using these weight windows should improve the computational performance of VERA as applied to ex-core calculations.

\subsection{FISSION SOURCE SPECTRUM}

Investigation of the fission source spectrum used in Shift was undertaken as part of this milestone effort to determine if a more detailed representation of the energy distribution was warranted, especially when considering detector response calculations. The following source spectrum options used for the fixed source in Shift are now available in VERA:

- ${ }^{235} \mathrm{U}$ Watt spectrum;

- nuclide-dependent Watt spectra based on ${ }^{235} \mathrm{U},{ }^{238} \mathrm{U},{ }^{239} \mathrm{Pu}$, and ${ }^{241} \mathrm{Pu}$; and

- MPACT multigroup spectrum.

Previously, the ${ }^{235} U$ Watt spectrum was the only source energy distribution available for fixed source calculations; however, the nuclide-dependent Watt spectra option is now the default to provide more fidelity. The MPACT multigroup spectrum will usually be the standard 51-group structure, and this nonseparable source will require significantly more memory compared to the other options. Full details about these options and their implementation can be found in the PHYSOR 2020 article from Davidson et al. [3].

\subsection{THREADING}

The integration of multi-threaded Shift into VERA was completed under milestone L2.RTM.MCH.P19.01, and full details can be found in the corresponding milestone report (CASL-U-2020-1954-000). Only the MC transport solve in Shift is threaded, and this is what is used by VERA. The user only needs to specify the num_threads option in the Shift block of the VERA input to turn on this feature, assuming the executable was built with OpenMP enabled. There are a few caveats to keep in mind when using the threading implementation:

- Only the Shift transport part of the calculation is threaded; MPACT has multithreading implemented but does not scale well beyond 4 threads per processor, so parallel decomposition is the preferred method for computational performance; COBRA-TF (CTF) is not threaded.

- The user should take care to run calculations with the appropriate message passing interface (MPI) options to see the full benefits of running with threads. 
- Threading cannot be used simultaneously with domain decomposition in Shift.

Threading enables Shift to run with fewer replicated processes on a node while still using the other processors for $\mathrm{MC}$ transport. This provides a means to significantly reduce memory usage while maintaining the $\mathrm{MC}$ transport time nearly constant. 


\section{RESULTS}

This section presents the test results of the methods outlined in Section 2, including the results of running a subcritical multiplication full core ex-core calculation with VERA.

\subsection{FISSION SOURCE SPECTRUM}

Full details on the investigation of the fission source spectrum used in Shift for ex-core calculations can be found in a previously published conference paper [3]. The main conclusions from this investigation were that the 51-energy group spectrum from MPACT is not useful because it is targeted for thermal calculations, and ex-core quantities are determined by faster energies. There is no discernible effect on relative detector response between using the ${ }^{235} \mathrm{U}$ Watt spectrum and the nuclide-dependent Watt spectra. For vessel fluence calculations, the nuclide-dependent Watt spectra should be used by Shift to account for burned fuel, especially on the periphery of the core.

\subsection{THREADING}

Full details on the memory savings and computational performance of threaded ex-core calculations with VERA are given in the L2.RTM.MCH.P19.01 milestone report (CASL-U-2020-1954-000). This section details the important parameters, memory usage, and computational performance applied to a subcritical multiplication, full core, detector response calculation for Watts Bar Nuclear Plant Unit 1 (WBN1).

A subcritical WBN1 ex-core model that was developed for validating source range detector responses during refueling as detailed in Godfrey et al. [4] was used to demonstrate the completion of this milestone. The specific model run was for startup of cycle 8 during the last step of refueling, and it had a single state point and no thermal-hydraulic feedback. The ex-core model includes the following details:

- 8 power range detectors (top and bottom),

- 2 source range detectors (intermediate range detectors not modeled),

- 2 dual surveillance capsules with no coupons (northeast and southwest quadrants),

- 2 single surveillance capsules (northwest and southeast quadrants), and

- a cylindrical concrete shield with detector wells.

A raytrace of the model geometry near the core mid-plane is given in Fig. 1. The flux and ${ }^{235} \mathrm{U}$ response were tallied in both source range detectors and all 8 power range detectors. The problem was run with CADIS variance reduction and optimized for the flux in the north and south source range detectors. An axial slice through the resulting adjoint function is shown in Fig. 2. VERA runs MPACT and then transfers the fission source distribution to Shift for MC transport. As discussed in Section 2.2, the nuclide-dependent Watt spectra for ${ }^{235} \mathrm{U},{ }^{238} \mathrm{U},{ }^{239} \mathrm{Pu}$, and ${ }^{241} \mathrm{Pu}$ can now be transferred to Shift from MPACT. This new feature was used for these calculations, and the normalized spatial distributions of the nuclide sources are given in Fig. 3. As shown in these figures, the locations of the startup source can be seen in the north and south locations at the end of refueling. 


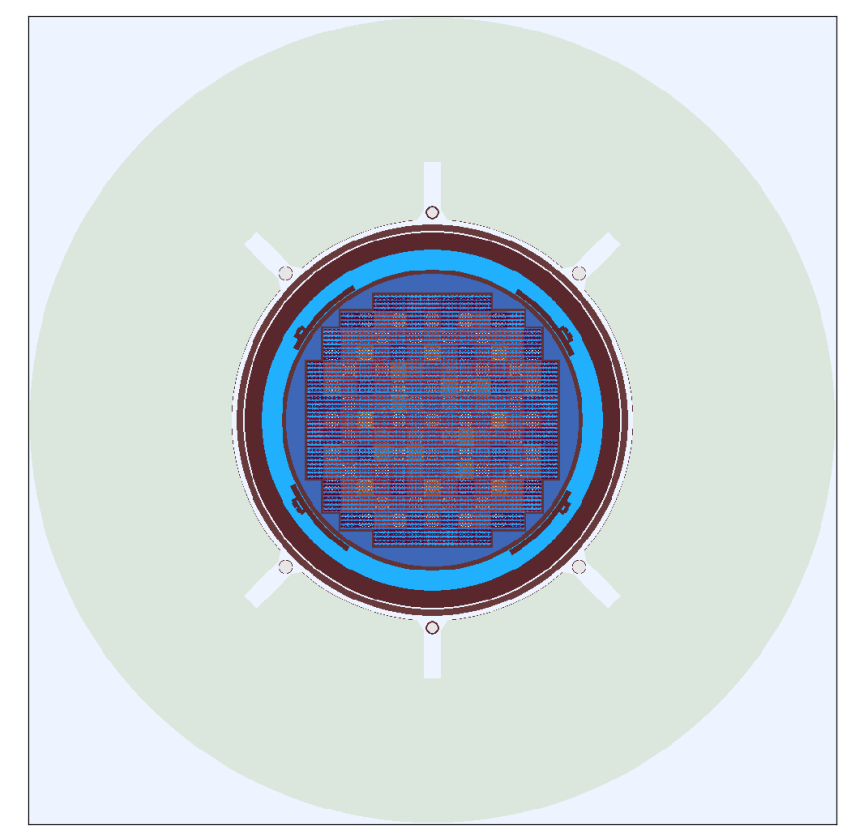

Figure 1. Axial slice of the WBN1 model at an elevation of $200 \mathrm{~cm}$. The colors represent the materials in each region.

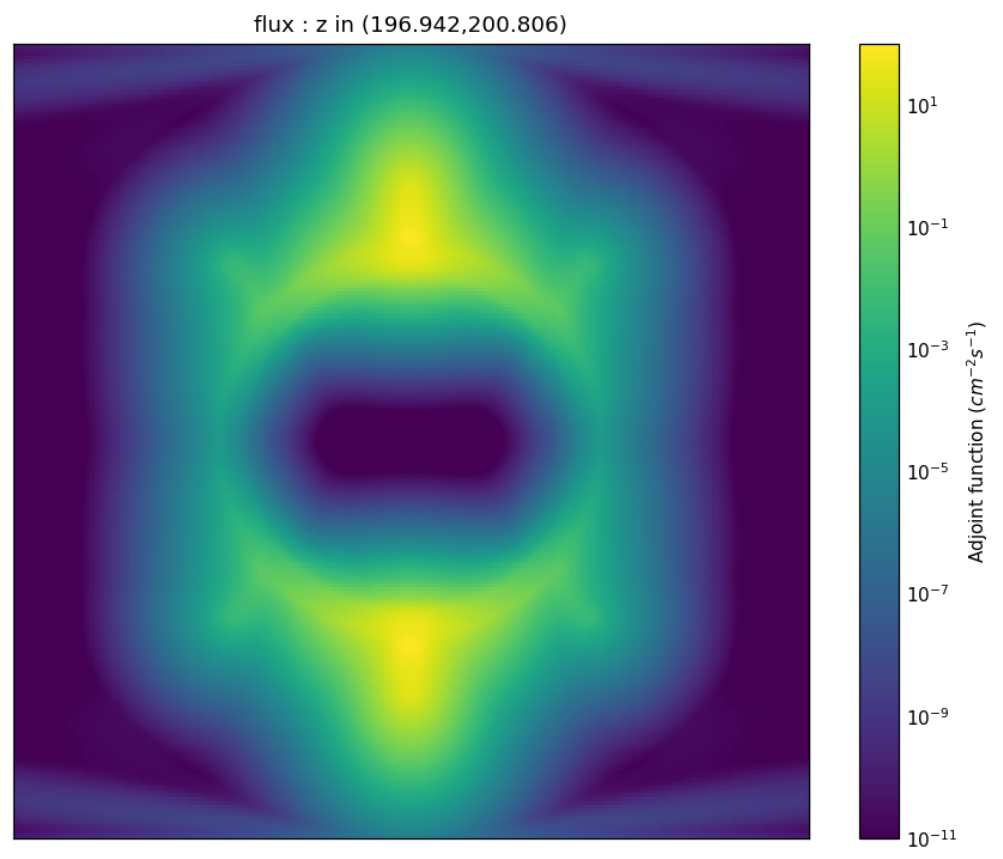

Figure 2. Axial slice of the WBN1 adjoint function for the north and south source range detectors at an elevation of $200 \mathrm{~cm}$. 


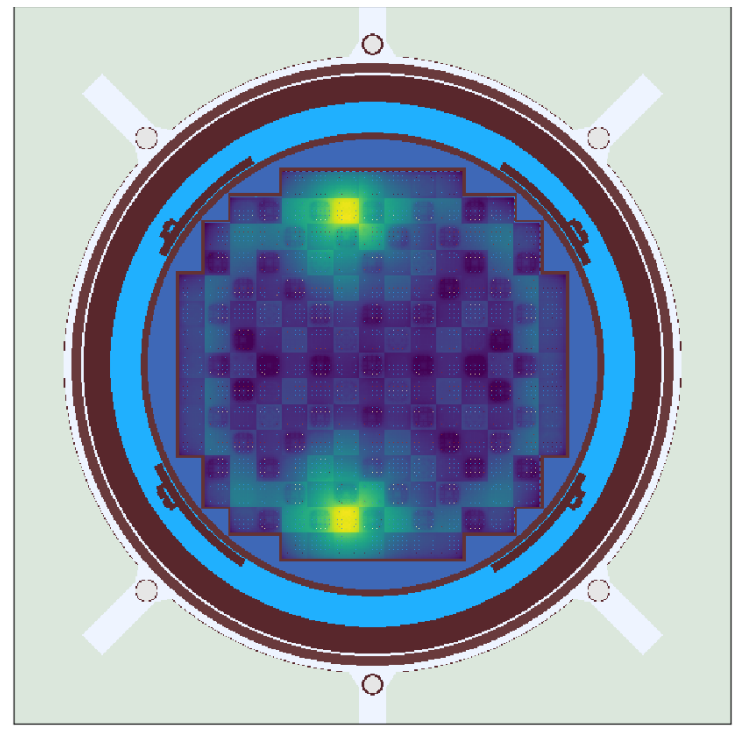

(a) ${ }^{235} U$ source

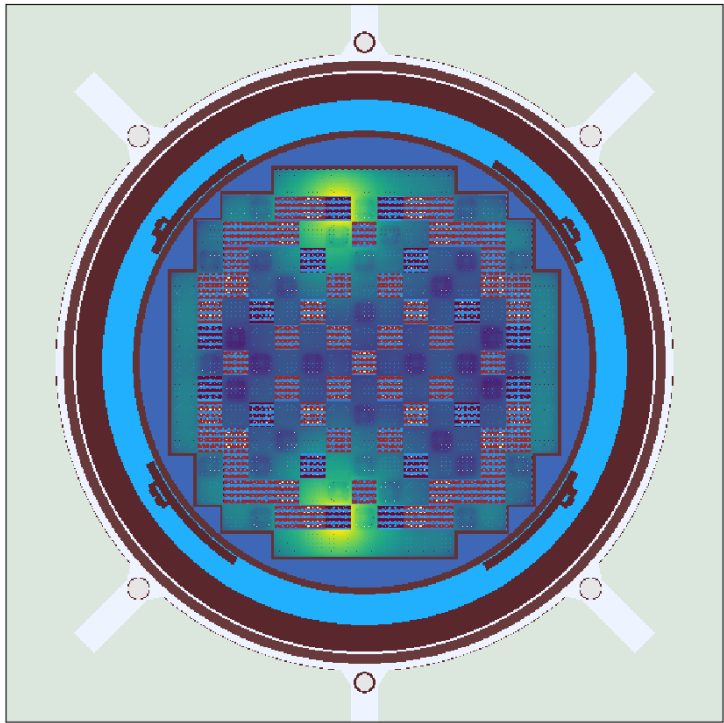

(c) ${ }^{239} \mathrm{Pu}$ source

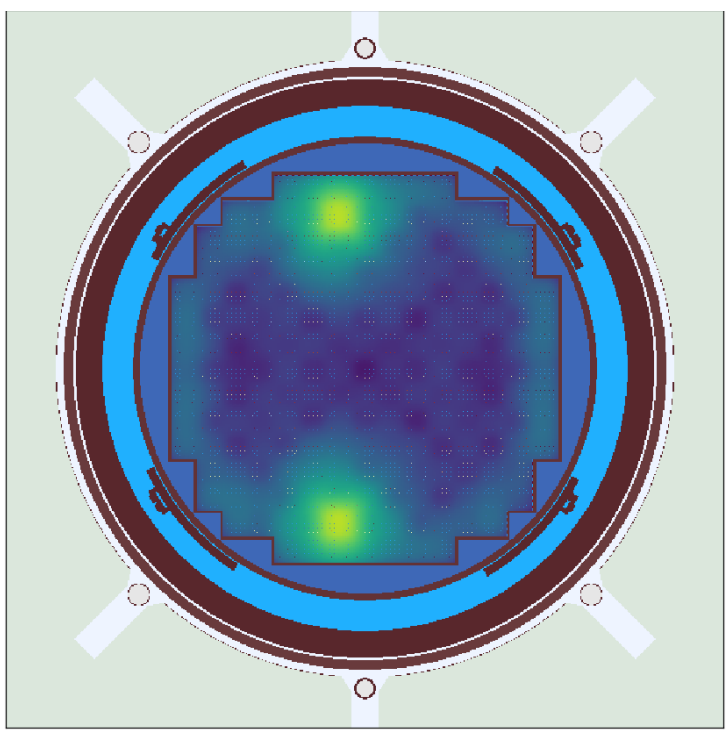

(b) ${ }^{238} U$ source

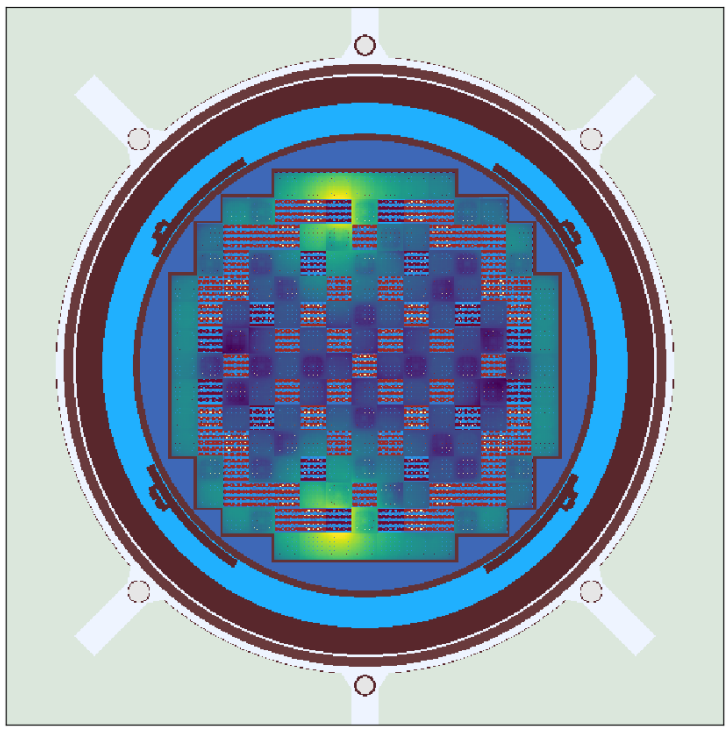

(d) ${ }^{241} P u$ source

Figure 3. Spatial distributions of the nuclide sources transferred from MPACT to Shift. Each source uses the appropriate Watt spectrum for the energy distribution.

The subcritical multiplication calculation was run on the Compute and Data Environment for Science (CADES) Oak Ridge National Laboratory (ORNL) institutional cluster. CADES is a commodity Intel x86_64 cluster with 32 cores and 128 GB of RAM per node. Due to its large size, this problem cannot fit in memory with 32 MPI tasks per node. This provided a good opportunity to run with threading to better utilize the nodes. The problem was run on 115 nodes with 16 cores 
per node for a total of 1,840 cores. The MPACT solution converged with default parameters on 90 nodes or 1,440 cores. The Shift MC calculation ran on 25 nodes with 400 MPI tasks and 2 threads per task. This configuration enabled Shift to fully utilize the available cores on the node during the MC transport step of the calculation.

The total calculation time was $5.87 \mathrm{E}+03 \mathrm{sec}$, with Shift transporting $1.0 \mathrm{E}+08$ particles. The computation times for different stages of the calculation are given in Table 1 . The total memory used across the Shift nodes was 2,021 GB for an average of 5 GB per core. Table 1 indicates that the fraction of time used to complete the MC transport calculation is small compared to the full computation time. The resulting tallies in the source range detectors have statistical uncertainties of $5 \%$ or less in the flux and ${ }^{235} \mathrm{U}$ response.

Table 1. Computation time for WBN1 full core subcritical model.

\begin{tabular}{lcc}
\hline $\begin{array}{l}\text { Calculation } \\
\text { component }\end{array}$ & $\begin{array}{c}\text { Computation } \\
\text { time }(\mathbf{s})\end{array}$ & $\begin{array}{c}\text { Fraction } \\
\text { of total }\end{array}$ \\
\hline Total & $5.87 \mathrm{E}+03$ & - \\
MPACT solve & $4.63 \mathrm{E}+03$ & 0.79 \\
Shift execution & $9.23 \mathrm{E}+02$ & 0.16 \\
Shift transport & $4.92 \mathrm{E}+02$ & 0.08 \\
\hline
\end{tabular}




\section{SUMMARY}

The ability to run fully loaded full core subcritical ex-core calculations with VERA has been implemented by employing multithreading. This ability has been demonstrated on a startup source range detector response calculation of a WBN1 model. Additionally, full source biasing and separable weight windows from Shift have been integrated into VERA, as well as the ability to transfer a nuclide-dependent fission source or an MPACT multigroup fission source spectrum.

\subsection{FUTURE}

Improvements and the addition of features for running full core subcritical multiplication ex-core calculations will continue under the VERA User's Group. 


\section{ACKNOWLEDGMENTS}

The authors would like to acknowledge the invaluable assistance from the following individuals in completing this milestone:

- Eva Davidson

- Cole Gentry

- Gregory Davidson

- Thomas Evans

- Shane Stimpson

- Shane Henderson

- Mark Baird

Furthermore, the authors would like to thank all of the MPACT and Shift developers for their collaboration on completing this milestone. Finally, the authors would like to acknowledge and thank Gary Wolfram and Tennessee Valley Authority (TVA) for providing the data needed for the WBN1 model.

This work was supported by the Consortium for Advanced Simulation of Light Water Reactors (http://www.casl.gov), an Energy Innovation Hub (http://www.energy.gov/hubs) for Modeling and Simulation of Nuclear Reactors under US Department of Energy (DOE) Contract No. DE-AC0500OR22725. This research used resources of the Compute and Data Environment for Science (CADES) at the Oak Ridge National Laboratory, which is supported by the Office of Science of the US Department of Energy under Contract No. DE-AC05-00OR22725.

\section{REFERENCES}

[1] J. C. Wagner and A. Haghighat. Automated variance reduction of Monte Carlo shielding calculations using the discrete ordinates adjoint function. Nuclear Science and Engineering, 128(2):186-208, 1998.

[2] E. D. Biondo, T. M. Evans, G. G. Davidson, and S. P. Hamilton. Singular value decomposition of adjoint flux distributions for Monte Carlo variance reduction. Annals of Nuclear Energy, 141, June 2020.

[3] E. E. Davidson, T. M. Pandya, K. E. Royston, T. M. Evans, A. T. Godfrey, and S. C. Henderson. Effect of fission source spectrum on Monte Carlo calculation of ex-core quantities. In Proc. PHYSOR 2020. American Nuclear Society, 2020.

[4] A. T. Godfrey, E. E. Davidson, G. Wolfram, B. Collins, C. Gentry, G. Ilas, S. Palmtag, T. Pandya, and K. Royston. Watts Bar Unit 1 source range detector response validation during refueling. Technical Report CASL-U-2018-1561-000, CASL, 2018. 